\title{
The Influence of Age on Cardiovascular, Motor, and Lifestyle Components in Hispanic-Latinos Living with HIV
}

Martín G. Rosario PT*, PhD, CSFI, ATRIC, Elizabeth Orozco, PT, DPT

Texas Woman's University, Physical Therapy Program, Dallas Campus; Texas, United States.

\author{
Article Details \\ Article Type: Research Article \\ Received date: $16^{\text {th }}$ October, 2021 \\ Accepted date: $09^{\text {th }}$ December, 2021 \\ Published date: $11^{\text {th }}$ December, 2021
}

Corresponding Author: Martín G. Rosario PT, PhD, CSFI, ATRIC, Assistant Professor, Texas Woman's University, Physical Therapy Program, Dallas Campus, 5500 Southwestern Medical Ave. Dallas, TX 75235-7299. United States. E-mail: mrosario1@twu.edu

Citation: Rosario, M.G. \& Orozco, E., (2021). The Influence of Age on Cardiovascular, Motor, and Lifestyle Components in Hispanic-Latinos Living with HIV. J Pub Health Issue Pract 5(2): 190. doi: https://doi.org/10.33790/jphip1100190

Copyright: (C2021, This is an open-access article distributed under the terms of the Creative Commons Attribution License 4.0, which permits unrestricted use, distribution, and reproduction in any medium, provided the original author and source are credited.

\begin{abstract}
Background: People living with HIV experience various comorbidities associated with aging. As this population grows older, these comorbidities cause physical impairments that decrease functional autonomy and quality of life. Purpose: This investigation aims to characterize various components affecting Hispanic Latino people living with HIV (PLHIV) concerning age.

Methods: Data was extracted from 323 participants' records enrolled in La Perla de Gran Precio's HIV Community Center in Puerto Rico. Participants were allocated into the following age groups for comparison: 40 years or less (L-HIV group) and above 40 years of age (U-HIV group). Multiple one-way ANOVAs were applied to compare the data between the two age groups based on five components: cardio, motor, lipid panel, comorbidities, and lifestyle.

Results: There were 44 participants allocated to the L-HIV group (age $=35.4+/-3.7$ ) and 279 to the U-HIV group (age=56.3+/-7.4). The cardio components (time completed on the submaximal treadmill test and peak heart rate) were significantly less in the upper age group. The U-HIV group also demonstrated substantially slower gait speed and less inclination achieved on the submaximal treadmill test. Lastly, the U-HIV group consisted of more participants with multiple comorbidities.
\end{abstract}

Conclusion: In Hispanic Latino PLHIV, more significant cardiovascular and motor deficits were identified in the U-HIV group in addition to an increased rate of multimorbidity. Future inquiries are desired to resolve if the time since diagnosis is an acceptable indicator of cardio-motor deterioration and an upsurge in multiple comorbidities experienced in this population.

\section{Introduction}

According to the HIV Surveillance Report published by the Centers for Disease Control and Prevention (CDC) in February 2019, the worldwide, 1.1 million of which are in the United States (US) alone. Within the US, Texas and Puerto Rico have the highest incidence rates, which have increased in the last few years. When considering age, young adults 25-34 years old contain the highest number of new HIV diagnoses, whereas those 50-54 years of age made up the largest percentage of people currently living with HIV [1].

With the advancements in antiretroviral therapy (ART), people with HIV are living longer and close to average life spans ( $>70$ years old). human immunodeficiency virus (HIV) affects 36.9 million people

However, increased life expectancy is accompanied by comorbidities associated with HIV infection, chronic inflammation, side effects of the antiretroviral medications, and lifestyle factors [2,3]. For instance, dyslipidemia and glucose abnormalities are typical following ART's long-term use [4,5]. Age-related illnesses such as cardiovascular deficiencies, kidney problems, liver disease, osteoporosis, muscle loss, and neurocognitive conditions also tend to emerge sooner in this population [6]. This multimorbidity negatively impacts the quality of life, physical activity levels, and functional independence in people living with HIV [7,8]. As a result, people living with HIV (PLHIV) experience a decline in cardiovascular function, muscle strength, and postural control, thus increasing their risk for falls and increasing the burden of care $[3,6,9]$. It is unclear whether the virus itself accelerates aging or if HIV is a risk factor for developing comorbidities associated with aging.

Therefore, the study's purpose was to describe the role age plays on cardiovascular and motor function, lipid profile, comorbidities, and different lifestyle components in Hispanic Latino PLHIV. It has been hypothesized that people living with HIV, 40 and older, will demonstrate a significantly greater decline in cardiovascular and motor function and higher comorbidities than younger individuals $(<40)$ living with HIV.

\section{Methods}

All participants were enlisted in a Community-Based Center (La Perla de Gran Precio) in San Juan, Puerto Rico, from 2000 to 2020 after signing approval by a Medical Doctor. LPGP is a community center dedicated to enhancing wellness and improving life quality through exercise therapy to Hispanic Latino PLHIV. This investigation was approved by the LPGP and adhered to the privacy and confidentiality standard established by the institution.

As an admission requirement, members signed an informed consent expressing their willingness to participate in this program. Additionally, participants submitted their most recent blood tests and completed various fitness tests. Information about lifestyle, comorbidities, CD4 count and lipid profile was gathered by interview and most current blood work. Further, cardiovascular and motor data were obtained with the completion of the Ross submaximal cardiovascular test.

First, data from all members enrolled in LPGP from 2000-2020 (approximately 1,300 files) were examined for data/information and completeness. This study recorded the most recent information 
available for all participants. Some of the information gathered included medications, years with HIV diagnosis, demographics (age, gender), HIV status (CD4 count), vitals, and physical activity status. Most of the participants at this HIV clinic were Hispanic/Latino (in the United States); thus, this project will give us a favorable profile of their ethnic group.

Second, the specific data collected was organized into three main components.

Motor and Cardiovascular components: The Ross submaximal test collected pre and post cardiovascular data (heart rate and blood pressure) and motor data (speed and treadmill inclination) while walking on a treadmill. As time progressed, the inclination and speed of the treadmill increased as well. The test was terminated when the maximal cardiac frequency was achieved or when participants reported the onset of muscle or cardiovascular fatigue. The cardiomotor data presented in this study represents the moment the Ross test ended. Data retrieved from participants' records were heart rate and blood pressure for cardiovascular components and speed and inclination of the treadmill for the motor components.

Lifestyle, comorbidities, lipids and immune components: Lifestyle and comorbidity information was gathered by an interview where participants were asked about their lifestyle habits (smoker, sedentary, etc.), and medical history was obtained. Additionally, the lipid profile and CD4 count were collected from the doctor's most recent blood work results and other notes.

Third, following data collection, a codebook was prepared for data mapping and comparisons. The codebook also provided data code and variable definition. The data was then organized in a database for further analysis, comparison, and interpretation.

\section{Data Analysis}

Data were organized into two groups by age. Participants 40 years of age or younger were allocated to the lower age group (L-HIV group). Subjects above 40 years of age were assigned to the upper age group (U-HIV group) for comparison.

Multiple one-way analysis of variances (ANOVA) with SPSS (25) was performed to compare both groups based on the following five components: cardiovascular, comorbidities, motor, lifestyle, and lipid profile.

\section{Results}

Data was collected from 323 participants' records. There were 44 participants assigned to the L-HIV group (age $=35.4+/-3.7$ ) and 279 to the U-HIV (age=56.3+/-7.4) groups. The L-HIV and $\mathrm{U}-\mathrm{HIV}$ were comparable in CD4 (L-HIV $=656.3+/-373.7$ and $\mathrm{U}-\mathrm{HIV}=619.0+/-346.6$ ), years of diagnosis $(\mathrm{L}-\mathrm{HIV}=20.98+/-8.73$ and U-HIV=19.84+/-8.42), and gender ratio, being mostly males (Table 1).

\begin{tabular}{|l|l|l|l|}
\hline Characteristics & $\leq 40$ years old & $>40$ years old & P-value \\
\hline Age & $\begin{array}{l}\mathrm{M}=35.39+/-3.69 \\
\text { years }\end{array}$ & $\begin{array}{l}\mathrm{M}=56.28+/-7.44 \\
\text { years }\end{array}$ & $\mathrm{P}=<.001$ \\
\hline Gender & $\begin{array}{l}\text { Male }=168 \\
\text { Female }=30 \\
\mathrm{M}=0.26+/-0.441\end{array}$ & $\begin{array}{l}\text { Male }=198 \\
\text { Female }=53 \\
\mathrm{M}=0.29+/-0.457\end{array}$ & $\mathrm{P}=.464$ \\
& $\mathrm{M}=14.09+/-6.51$ & $\mathrm{M}=20.12+/-8.714$ & $\mathrm{P}=<.001$ \\
\hline Time since Dx & $\mathrm{M}=656.30+/-$ & $\begin{array}{l}\mathrm{M}=619.02+/- \\
346.60\end{array}$ & $\mathrm{P}=.512$ \\
\hline Cd4 & 373.69 & \\
& Table 1: Demographic data of all participants \\
\hline
\end{tabular}

\section{Cardiovascular and Motor Components}

Cardiovascular components included the following: time completed during the treadmill test and vitals (heart rate and blood pressure) taken at the test's termination. Motor components consisted of speed and percent of inclination achieved during the treadmill test. Descriptive statistics for these variables were organized by the group and can be found in table 2. For the cardiovascular components, the
U-HIV group achieved a significantly lower heart rate $(p<.05)$ and completed less time $(\mathrm{p}<.05)$ on the submaximal treadmill test than the L-HIV group. The mean systolic and diastolic blood pressure were higher in the U-HIV; however, this finding was not significant. For the motor component, the U-HIV group demonstrated a significantly slower walking speed $(\mathrm{p}<.05)$ and achieved less inclination $(\mathrm{p}<.05)$ during the Ross test when compared to the L-HIV group.

\begin{tabular}{|l|l|l|l|l|}
\hline Characteristics & $\leq 40$ years old & $>40$ years old & F Value & $\mathrm{P}$-value \\
\hline Heart Rate (bpm) & $\begin{array}{l}\mathrm{M}=152.27+/- \\
16.50\end{array}$ & $\begin{array}{l}\mathrm{M}=136.63+/- \\
17.73\end{array}$ & 30.11 & $\mathrm{P}=<.001$ \\
\hline $\begin{array}{l}\text { Systolic BP } \\
(\mathrm{mmHg})\end{array}$ & $\begin{array}{l}\mathrm{M}=122.91+/- \\
12.94\end{array}$ & $\begin{array}{l}\mathrm{M}=124.32+/- \\
17.20\end{array}$ & 0.22 & $\mathrm{P}=.644$ \\
\hline $\begin{array}{l}\text { Diastolic BP } \\
(\mathrm{mmHg})\end{array}$ & $\begin{array}{l}\mathrm{M}=72.43+/- \\
9.14\end{array}$ & $\begin{array}{l}\mathrm{M}=75.25+/- \\
11.07\end{array}$ & 2.05 & $\mathrm{P}=.154$ \\
\hline Cardio test Time & $\begin{array}{l}\mathrm{M}=13.24+/- \\
4.21\end{array}$ & $\mathrm{M}=9.90+/-4.62$ & 20.30 & $\mathrm{P}=<.001$ \\
\hline Motor Component at the end of the Ross Submaximal Test & \\
\hline Velocity & $\begin{array}{l}\mathrm{M}=3.40+/- \\
.001\end{array}$ & $\mathrm{M}=3.30+/-.28$ & 5.68 & $\mathrm{P}=.018$ \\
\hline Inclination & $\begin{array}{l}\mathrm{M}=8.05+/- \\
4.38\end{array}$ & $\begin{array}{l}\mathrm{M}=5.12+/- \\
4.02\end{array}$ & 19.64 & $\mathrm{P}=<.001$ \\
\hline
\end{tabular}

Table 2 Cardiovascular Component at the end of the Ross Submaximal Test 
Comorbidity, Lipid, and Lifestyle Components: Table 3 illustrates the ten most common comorbidities reported by this study's participants. Although not significant, the U-HIV group was more likely to experience these comorbidities than the L-HIV group.

The lipid component (Table 3) represents the specific lipid values of all participants. While not significant, results showed greater cholesterol, triglycerides, and VLDL values in the U-HIV group than L-HIV; however, the L-HIV demonstrated greater HDL values.
Data in table 3 also lists the main lifestyle habits reported by all participants. Among the five listed patterns and comorbidities, participants in the U-HIV exhibited a slight increase in many factors compared to L-HIV. However, the U-HIV group reported having significantly $(\mathrm{P}<0.05)$ more comorbidities than the L-HIV. Further, the U-HIV group's number of ART medications was slightly lower than the L-HIV group.

\begin{tabular}{|c|c|c|c|c|}
\hline Characteristics & $\leq 40$ years old & $>40$ years old & F Value & P-value \\
\hline High Blood Pressure & $\mathrm{M}=0.86+/-2.61$ & $\mathrm{M}=1.16+/-2.66$ & 0.49 & $\mathrm{P}=.484$ \\
\hline Chronic Pain & $\mathrm{M}=0.86+/-2.61$ & $\mathrm{M}=1.04+/-2.69$ & 0.16 & $\mathrm{P}=.686$ \\
\hline Neuropathy & $\mathrm{M}=0.86+/-2.61$ & $\mathrm{M}=1.13+/-2.67$ & 0.39 & $\mathrm{P}=.533$ \\
\hline Depression & $\mathrm{M}=0.98+/-2.59$ & $\mathrm{M}=1.13+/-2.67$ & 0.12 & $\mathrm{P}=.725$ \\
\hline Diarrhea & $\mathrm{M}=0.84+/-2.61$ & $\mathrm{M}=0.94+/-2.70$ & 0.05 & $\mathrm{P}=.829$ \\
\hline Hiperlipidemia & $\mathrm{M}=0.82+/-2.62$ & $\mathrm{M}=1.13+/-2.67$ & 0.51 & $\mathrm{P}=.477$ \\
\hline Fatigue & $\mathrm{M}=0.84+/-2.61$ & $\mathrm{M}=.96+/-2.70$ & 0.08 & $\mathrm{P}=.778$ \\
\hline Arthritis & $\mathrm{M}=0.82+/-2.62$ & $\mathrm{M}=0.94+/-2.70$ & 0.07 & $\mathrm{P}=.788$ \\
\hline Anemia & $\mathrm{M}=0.84+/-2.61$ & $\mathrm{M}=0.93+/-2.71$ & 0.04 & $\mathrm{P}=.842$ \\
\hline Diabetes & $\mathrm{M}=0.82+/-2.62$ & $\mathrm{M}=0.97+/-2.70$ & 0.13 & $\mathrm{P}=.719$ \\
\hline Others Dx & $\mathrm{M}=1.16+/-2.86$ & $\mathrm{M}=1.09+/-2.67$ & 0.02 & $\mathrm{P}=.880$ \\
\hline \multicolumn{5}{|c|}{ Lipids Components Reported in the Lab analysis } \\
\hline \multirow[t]{2}{*}{ Characteristics } & $\leq 40$ years old & $>40$ years old & & \\
\hline & F value & P-value & & \\
\hline Cholesterol & $\begin{array}{l}\mathrm{M}=165.33+/- \\
38.90\end{array}$ & $\mathrm{M}=178.38+/-45.53$ & 2.35 & $\mathrm{P}=.127$ \\
\hline Triglyceride & $\begin{array}{l}M=148.73+/- \\
66.31\end{array}$ & $\begin{array}{l}M=182.73+/- \\
140.69\end{array}$ & 1.46 & $\mathrm{P}=.229$ \\
\hline HDL & $\begin{array}{l}\mathrm{M}=47.53+/- \\
31.95\end{array}$ & $\mathrm{M}=44.38+/-13.18$ & 0.67 & $\mathrm{P}=.414$ \\
\hline VLDL & $\begin{array}{l}\mathrm{M}=29.48+/- \\
6.67\end{array}$ & $M=39.67+/-32.74$ & 0.77 & $\mathrm{P}=.384$ \\
\hline \multicolumn{5}{|c|}{ Lifestyle Components Reported by Participants } \\
\hline Characteristics & $\leq 40$ years old & $>40$ years old & F value & P-value \\
\hline Over 50 years & $\mathrm{M}=0.84+/-2.61$ & $\mathrm{M}=1.14+/-2.66$ & 0.48 & $\mathrm{P}=.488$ \\
\hline Stress & $\mathrm{M}=0.93+/-2.60$ & $\mathrm{M}=1.03+/-2.69$ & 0.05 & $\mathrm{P}=.817$ \\
\hline Smoker & $\mathrm{M}=1.02+/-2.58$ & $\mathrm{M}=1.09+/-2.68$ & 0.02 & $\mathrm{P}=.884$ \\
\hline Obese & $\mathrm{M}=0.91+/-2.60$ & $\mathrm{M}=1.03+/-2.69$ & 0.76 & $\mathrm{P}=.783$ \\
\hline Sedentary & $\mathrm{M}=0.91+/-2.60$ & $\mathrm{M}=1.04+/-2.69$ & 0.09 & $\mathrm{P}=.770$ \\
\hline $\begin{array}{l}\text { MC-Multiple } \\
\text { Comorbidities }\end{array}$ & $\mathrm{M}=1.02+/-2.02$ & $\mathrm{M}=2.14+/-2.65$ & 7.20 & $\mathrm{P}<0.05$ \\
\hline $\begin{array}{l}\text { Meds- Multiple } \\
\text { Meds }\end{array}$ & $\mathrm{M}=4.14+/-3.47$ & $\mathrm{M}=3.60+/-3.01$ & 1.15 & $\mathrm{P}=.284$ \\
\hline
\end{tabular}

\section{Discussion}

This study intended to examine and characterize the various physical profiles concerning age and the disease's progression in Hispanic Latino PLHIV. In pursuit of investigating this difference, our study analyzes the connection between the cardio-motor, lipid panel, comorbidities, and lifestyle components, which are essential physical fitness factors, regarding subjects' immune profiles (CD4 values) and quality of life in Hispanic Latinos living with HIV. To verify the above, this endeavor distributes age into two brackets, low age group: $<40$ years old and, and upper age group, $>40$ years old.
Cardio components: This study further highlights that people living with HIV demonstrate deficits in cardiovascular function, as evident by a decline in cardiorespiratory fitness (CRF). It is known that cardiac dysfunction frequently occurs in PLHIV. In fact, people living with HIV are 1.5-2 times more likely to develop cardiovascular disease (CVD) compared to people without HIV [10]. Cardiorespiratory fitness is a crucial predictor of CVD. People living with HIV have reduced cardiorespiratory fitness, and age has been shown to be a moderator in CRF in this population [3].

In 2006, Oursler et al. [11] utilized a submaximal test known as the modified Bruce treadmill test to identify differences in the aerobic 
capacity measured by VO2 peak when considering age. They found that the older group of $\mathrm{HIV}^{+}$men (50-59 years old) demonstrated a $26 \%$ reduction in aerobic capacity when compared to the younger group (40-49 years old) [11]. Another study by Lorenzo et al. [12] also utilized the modified Bruce protocol to assess cardiac risk factors in this population, and they found an inverse relationship between VO2 peak and age. While neither study compared the time completed on the modified Bruce treadmill test with age, it can be implied that a lower $\mathrm{VO} 2$ peak would be associated with less time completed on the submaximal test.

Although our study used time completed on the Ross test to measure cardiorespiratory fitness, our findings are consistent with previous literature. The upper age group demonstrates a significant decline in cardiorespiratory fitness compared to the lower age group. This study also revealed that the upper age group demonstrated a significant decrease in peak HR achieved during the Ross submaximal test compared to the lower age group. This decrease in HR could have occurred for the following two reasons: the test was terminated once the participant reached his age-predicted max heart rate (which could have occurred a lot sooner in the upper age group than the lower age group). Alternatively, the participant requested to terminate the test before reaching the max heart rate secondary to muscular or cardiovascular fatigue.

Furthermore, regular participation in aerobic exercise has been shown to improve cardiorespiratory fitness in this population and decrease the risk of developing CVD. In fact, it has been recommended that people living with HIV participate in aerobic exercise or a combination of aerobic exercise plus resistance training at least three times a week to maintain and improve cardiovascular health [13].

One shortcoming to this study is that the cardio data only contains measurements from a single point in time; therefore, we are unable to assess the rate at which cardiac function declines in this population. Further, there was no control group to compare our findings. Therefore, a longitudinal study is needed to understand better the decline in cardiorespiratory fitness over time and how it compares to HIV- age-matched controls.

Motor Components: In addition to the decline in cardiovascular health, this study also highlights the decline in motor function in PLHIV consistent with age. This decline was evident by reducing the percent of inclination and gait speed during the upper age group's Ross test.

Since inclination increases with time completed on the Ross test, a lower inclination level is associated with the earlier termination submaximal test. An increase in inclination is also associated with the greater workload for the lower extremities musculature and the heart. It has already been established that this population experiences deficits in cardiovascular function, but they also demonstrate reduced motor output that is often conveyed as muscle weakness. This decline in muscle strength is secondary to factors such as the virus, side effects of ART, and an increase in sedentary behaviors [14]. HIV has been shown to alter oxygen kinetics by limiting the lower extremity muscles' withdrawal and oxygen use [15]. As a result, lactate increases in the muscles with activity and reduces motor output, ultimately causing a quicker onset of fatigue [14]. Raso et al. [14] found that muscle strength was positively correlated with peak $\mathrm{VO} 2$ in people living with HIV, indicating that those with less muscle strength also demonstrated a lower VO2 peak.

Besides, earlier studies that analyzed gait speed also showed similar findings in this population. Schrack et al. [16] assessed the rate at which gait speed declines over a six-year period in men who were 40 years or older and infected with HIV compared to HIV-negative men. They discovered that HIV positive participants had a $57 \%$ greater risk of developing clinically slower gait speed. Furthermore, slower gait speed was also considered a prognostic indicator of accelerated aging and a faster functional decline rate in this population. When considering age, men 40-49 years old demonstrated a decline in gait speed regardless of HIV status. However, there was a significant difference between gait speeds among the two groups after the age of 50 . Schrack and colleagues found that on average, gait speed declined $0.025 \mathrm{~m} / \mathrm{s}$ more per year in men over the age of 50 who were $\mathrm{HIV}^{+}$ than those who were HIV-. Also, there was a significant interaction between age and HIV status, indicating that the groups' differences increased with age [16].

To our knowledge, this was the first inquiry comparing the difference in gait speed and the percent of inclination achieved during a submaximal test in PLHIV. Our examination is consistent with previous studies that PLHIV demonstrates more significant locomotor deficits over time [17]. These locomotor deficits could be due to more prolonged exposure to the virus and side effects of ART that have contributed to a decline in muscle strength and functional mobility. Like aerobic exercise, participation in progressive resistance exercise training at least three times a week has been recommended for this population to improve muscle strength [13]. In addition, assessments of strength and gait speed should be considered when treating individuals with HIV to understand their locomotor function better.

A deficiency of this work is that we did not objectively measure lower extremity strength or function. Therefore, we could not directly determine if reduced muscle strength or activation was associated with an inability to perform at greater inclination on the treadmill test. Future studies are required to determine if muscle strength and function are associated with percent of inclination or gait speed achieved during a submaximal treadmill test.

Comorbidity Components: This examination features the common comorbidities reported by Hispanic-Latinos living with HIV amongst the two age groups. Our results indicate that the U-HIV had an increased rate of all the comorbidities related to their counterparts. High blood pressure, neuropathy, depression, hyperlipidemia, and chronic pain are among the comorbidities with higher occurrences. Similar to our discoveries, previous reports [9] remarked that PLHIV presents with considerably increased rates of depression. However, literature has also confirmed that dementia, anxiety, and arthritis are other conditions heightened by HIV [9]

Our results suggest that regardless of age, both groups displayed comorbidities; however, the U-HIV presented a greater prevalence of comorbidities. Previous studies have determined a relationship between several ART medications and hyperlipidemia accompanied by pharmacological therapy's urgency to reduce the effects on health in PLHIV [4,5].

Chronic pain is another comorbidity that appears to be more prevalent in the U-HIV than the L-HIV group. Evidence proposes that more than half of the people living with HIV will experience chronic pain in their lifetime [18]. As a result, chronic pain will reduce the quality of life and increase disability in this community. Also, persistent pain has been associated with greater mental health disorders, leading to higher opioid use and increased suicidal rates than their HIV- counterparts $[18,19]$. These previous statements could explain why our U-HIV group presented with a higher rate of depression than the L-HIV.

Most chronic pain encountered in PLHIV is associated with peripheral neuropathy (PN) secondary to the effects of ART and the virus [18]. Rosario et al. [20] reported PN's association with HIV disease among Hispanic-Latinos living with HIV. PN alters muscle activation, leading to postural instability in those suffering from this condition [21].

In the future, studies should investigate the correlation between HIV severity, time since diagnosis, years with chronic pain, pain management medications, type of ART, and PN medication. Further, longitudinal research is recommended to explore all aspects discussed in this study. 
Lipid Component: This study features the comparison between age groups and some risk factors associated with the U-HIV group, higher cholesterol, and HDL. Our results indicate the importance of routine lipid panel lab work regardless of the CD4 values and maturing. Since ART has been implemented to manage HIV, hyperlipidemia has been a burden and a potential complication to PLHIV. Nevertheless, age or period living with HIV is not a consideration affecting lipid analysis in physically active HIV people with healthy CD4 levels.

Previous studies emphasize the ramification of reducing some risk factors related to an unhealthy rise in the lipid profile. We point out various components, such as smoking and sedentary life, to avert further health problems, like coronary artery disease [22].

We propose two aspects that contributed to the parallels in the lipid profile of our participants. First, the medication to treat hyperlipidemia had proven effective with lifestyle modifications such as aerobic exercise, a good diet, and smoking cessation [5]. Although we tallied specific participants taking lipid medications, we failed to compile the type of drug and dosage, making this lack of information a limitation of this study. Further research should consider the impact of various drugs and dosages on the lipid profile in PLHIV.

The second factor that might justify the similarities in our study's lipid profile is physical activity. Most of the participants of this investigation engaged in regular cardiovascular exercise. The LDGP community center has a gym with a personal trainer whose purpose is to provide a personalized fitness program for each participant. This fitness program is a requirement to be part of LDGP. Recently, Orozco and Rosario [23] described the advances of this particular LDGP fitness program. The authors emphasized the benefits of partaking in this program, especially on the immune system (CD4 count). The ongoing study, among other aspects, highlighted the positive implications of physical activity on a stable lipid panel, regardless of the side effects of ART in this population. We ushered in adding to the profile of those living with HIV previously established in Orozco and Rosario's [23] work.

To better understand the health profile of an individual living with HIV, we recommend assessing body composition (i.e., body mass index) to determine body fat distribution across the participant's life. The lack of body composition data is one crucial limitation of this study. Since this was a retrospective study, and body composition measurements were not obtained, body composition analyses were not performed. BMI profile is an essential factor to consider when treating PLHIV. Similar to our point of view, a systematic review by Berner et al. [6] revealed that obesity was correlated with poor dynamic balance, and disease severity showed conflicting associations with gait execution. Bauer et al. [24] identified an interrelationship between HIV status and BMI measurements. They associated obesity with further impairments on various balance and gait analyses. This study's clinical implication is noteworthy in that as people with HIV follow the national trend towards obesity, their risk for balance, and gait impairments substantially increase [24].

Lifestyle Component: This study highlights the distinctions in lifestyle components between the different age groups while considering comparable immune system status in HIV+ individuals. Among the variations attributed to lifestyle, smoking was documented considerably more in the U-HIV. Additionally, the U-HIV group exhibited multiple comorbidities compared to the L-HIV. This study's outcomes evoke that smoking might be associated with enduring multiple comorbidities later in life for our participants. Helleber M et al. [25] reported that HIV-infected individuals might lose more years of life by smoking than the virus itself. Increased mortality associated with smoking also increases markedly with age, and increased smoking-related mortality can be expected as the treated HIV-infected population ages.

It is well documented that life expectancy increases over 20 years with ART's acceptable use in PLHIV [26]. Nevertheless, smoking is reducing life expectancy even more so than the HIV disease in this population. LDGP encourages a healthy lifestyle and wellbeing, which might explain both groups' comparability regardless of the age difference. In agreement with Calza L et al. [5] and Sabin C.A. [27], we recognize lifestyle adjustments such as smoking cessation and regular exercise participation are necessary to improve quality of life and increase life expectancy.

The promotion of exercise and reasonable lifestyle choices are among the highest factors to avoid further complications in PLHIV. Among depressed adults, physical inactivity and smoking were strongly associated with health-related quality of life, life expectancy, and quality-adjusted life expectancy. In contrast, obesity and heavy drinking were only weakly associated with these indices [28]. Therefore, we recommend looking further into how lifestyle components such as smoking, physical activity levels, and BMI relate to one another in this population. Both factors, smoking and physical inactivity are critical factors for health-related quality of life deteriorating, life expectancy reduction, and quality-adjusted life expectancy shifts in those living with HIV [28].

The current work further illustrates the comparability of Hispanic Latino people living with HIV by age when considering lifestyle parameters. Another point to consider is that the older group experienced more comorbidities than the younger group. However, to better understand this, finding a thorough background screening of the participant's medical history is recommended.

The outcomes presented in this study were from the data collected from participants' records. As a result, there were constraints on the type of data collected. For instance, we were unable to gather information regarding the time of infection, the initiation of ART, the participant's adherence to ART, mortality rates, and life expectancy. Therefore, we suggest collecting the data as mentioned above for a more comprehensive assessment of the impact lifestyle choices have on life expectancy in Hispanic-Latinos living with HIV.

\section{Conclusion}

In all, our findings revealed a reduction in cardiovascular and motor function and a higher rate of comorbidities in the upper age group of people living with HIV. These findings imply that as this population ages, they are more likely to present with multimorbidities, leading to greater functional decline. However, it remains unclear how quickly cardio and motor function decline in this population and whether or not the time since diagnosis further contributes to the decline. Therefore, future research is needed to ascertain whether the time since diagnosis plays a greater role in the cardio-motor decline and increases the rate of multimorbidities experienced in PLHIV. The cardiovascular and neuromuscular function should be assessed regularly by clinicians working with PLHIV as impairments to these systems can negatively impact balance and gait.

\section{Declaration:}

\section{Funding: Not Applicable}

Conflicts of interest: Authors report no conflict of interest.

Ethics approval: IRB approval TWU protocol \# 20092

Availability of data and material (data transparency)

\section{References}

1. Centers for Disease Control and Prevention. Estimated HIV incidence and prevalence in the United States, 2010-2016. HIV Surveillance Supplemental Report 2019;24(No. 1). http://www. cdc.gov/ hiv/library/reports/hiv-surveillance.html. Published February 2019. Accessed October 14, 2019.

2. Allavena, C., Hanf, M., Rey, D., Duvivier, C., BaniSadr, F., Poizot-Martin, I., Jacomet, C., Pugliese, P., Delobel, P., Katlama, C., Joly, V., Chidiac, C., Dournon, N., Merrien, D., May, T., Reynes, J., Gagneux-Brunon, A., Chirouze, C., Huleux, T., Cabié, A., ... Dat'AIDS study group (2018). Antiretroviral exposure and comorbidities in an aging HIV-infected population: The challenge of geriatric patients. PloS one, 13(9), e 0203895. https://doi.org/10.1371/journal.pone.0203895. 
3. Vancampfort, D., Mugisha, J., Rosenbaum, S., Firth, J., De Hert, M., Probst, M., \& Stubbs, B. (2016). Cardiorespiratory fitness levels and moderators in people with HIV: A systematic review and meta-analysis. Preventive medicine, 93, 106-114. https:// doi.org/10.1016/j.ypmed.2016.10.001.

4. Calza, L., Manfredi, R., \& Chiodo, F. (2004). Dyslipidaemia associated with antiretroviral therapy in HIV-infected patients. The Journal of antimicrobial chemotherapy, 53(1), 10-14. https://doi.org/10.1093/jac/dkh013.

5. Calza, L., Manfredi, R., Pocaterra, D., \& Chiodo, F. (2008). Risk of premature atherosclerosis and ischemic heart disease associated with HIV infection and antiretroviral therapy. The Journal of infection, 57(1), 16-32. https://doi.org/10.1016/j. jinf.2008.02.006.

6. Berner, K., Morris, L., Baumeister, J., \& Louw, Q. (2017). Objective impairments of gait and balance in adults living with HIV-1 infection: a systematic review and meta-analysis of observational studies. BMC musculoskeletal disorders, 18(1), 325. https://doi.org/10.1186/s12891-017-1682-2doi:10.1186/ s12891-017-1682-2.

7. Lorenc, A., Ananthavarathan, P., Lorigan, J., Jowata, M., Brook, G., \& Banarsee, R. (2014). The prevalence of comorbidities among people living with HIV in Brent: a diverse London Borough. London journal of primary care, 6(4), 84-90. https:// doi.org/10.1080/17571472.2014.11493422.

8. Erlandson, K. M., Allshouse, A. A., Jankowski, C. M., Duong, S., Mawhinney, S., Kohrt, W. M., \& Campbell, T. B. (2012). Comparison of functional status instruments in HIV-infected adults on effective antiretroviral therapy. HIV clinical trials, 13(6), 324-334. https://doi.org/10.1310/hot1306-324.

9. Banks, L. M., Zuurmond, M., Ferrand, R., \& Kuper, H. (2015). The relationship between HIV and prevalence of disabilities in sub-Saharan Africa: systematic review (FA). Tropical medicine \& international health : TM \& IH, 20(4), 411-429. https://doi. org/10.1111/tmi.12449.

10. Lacson, J. C., Barnes, R. P., \& Bahrami, H. (2017). Coronary Artery Disease in HIV-Infected Patients: Downside of Living Longer. Current atherosclerosis reports, 19(4), 18. https://doi. org/10.1007/s11883-017-0651-4

11. Oursler, K. K., Sorkin, J. D., Smith, B. A., \& Katzel, L. I. (2006). Reduced aerobic capacity and physical functioning in older HIV-infected men. AIDS research and human retroviruses, 22(11), 1113-1121. https://doi.org/10.1089/aid.2006.22.1113.

12. Lorenzo, A. D., Meirelles, V., Vilela, F., \& Souza, F. C. C. (2013). Use of the exercise treadmill test for the assessment of cardiac risk markers in adults infected with HIV. Journal of the international association of providers of AIDS care (JIAPAC), 110-116. https://doi.org/10.1177/1545109712460098.

13. O'Brien, K. K., Tynan, A. M., Nixon, S. A., \& Glazier, R. H. (2017). Effectiveness of progressive resistive exercise (PRE) in the context of HIV: systematic review and meta-analysis using the Cochrane Collaboration protocol. BMC infectious diseases, 17(1)268. https://doi.org/10.1186/s12879-017-2342-8.

14. Raso, V., Shephard, R. J., Casseb, J., Duarte, A. J., Silva, P. R., \& Greve, J. M. (2013). Association between muscle strength and the cardiopulmonary status of individuals living with HIV/ AIDS. Clinics (Sao Paulo, Brazil), 68(3), 359-364. https://doi. org/10.6061/clinics/2013(03)oa12

15. Cade, W. T., Fantry, L. E., Nabar, S. R., Shaw, D. K., \& Keyser, R. E. (2003). Impaired oxygen on-kinetics in persons with human immunodeficiency virus are not due to highly active antiretroviral therapy. Archives of physical medicine and rehabilitation, 84(12), 1831-1838. https://doi.org/10.1016/j. apmr.2003.03.004.

J Pub Health Issue Pract

Volume 5. 2021. 190
16. Schrack, J. A., Althoff, K. N., Jacobson, L. P., Erlandson, K. M., Jamieson, B. D., Koletar, S. L., Phair, J., Ferrucci, L., Brown, T. T., Margolick, J. B., \& Multicenter AIDS Cohort Study (2015). Accelerated Longitudinal Gait Speed Decline in HIVInfected Older Men. Journal of acquired immune deficiency syndromes (1999), 70(4), 370-376. https://doi.org/10.1097/ QAI.0000000000000731.

17. Richert, L., Brault, M., Mercié, P., Dauchy, F. A., Bruyand, M., Greib, C., Dabis, F., Bonnet, F., Chêne, G., Dehail, P., \& Groupe d'Epidémiologie Clinique du SIDA en Aquitaine (GECSA) (2014). Decline in locomotor functions over time in HIVinfected patients. AIDS (London, England), 28(10), 1441-1449. https://doi.org/10.1097/QAD.00000000000.

18. Madden, V. J., Parker, R., \& Goodin, B. R. (2020). Chronic pain in people with HIV: a common comorbidity and threat to quality of life. Pain management, 10(4), 253-260. https://doi. org/10.2217/pmt-2020-0004.

19. Addis, D. R., DeBerry, J. J., \& Aggarwal, S. (2020). Chronic Pain in HIV. Molecular pain, 16, 1744806920927276. https:// doi.org/10.1177/1744806920927276.

20. Rosario M.G., Jamison L., \& Gines G. (2020). The Role of HIV Antiretroviral Medication on Motor-Cognitive and Neurological Alterations in Hispanic People Living with HIV. $J$ Pub Health Issue Pract 4(1):160. DOI: https://doi.org/10.33790/ jphip1100160

21. Trenkwalder, C., Straube, A., Paulus, W., Krafczyk, S., Schielke, E., \& Einhäupl, K. (1992). Postural imbalance: An early sign in HIV-1 infected patients. European archives of psychiatry and clinical neuroscience, 241(5), 267-272.

22. Falusi, O. M., \& Aberg, J. A. (2001). HIV and cardiovascular risk factors. The AIDS reader, 11(5), 263-268.

23. Orozco, E., \& Rosario, M.G. (2020). Overall fitness benefits in individuals with HIV participating in a community-based exercise program. J rehab Pract res 1(2):109. https://doi. org/10.33790/jrpr1100109

24. Bauer, L. O., Wu, Z., \& Wolfson, L. I. (2011). An obese body mass increases the adverse effects of HIV/AIDS on balance and gait. Physical therapy, 91(7), 1063-1071. https://doi. org/10.2522/ptj.20100292.

25. Helleberg, M., May, M. T., Ingle, S. M., Dabis, F., Reiss, P., Fätkenheuer, G., Costagliola, D., d'Arminio, A., Cavassini, M., Smith, C., Justice, A. C., Gill, J., Sterne, J. A., \& Obel, N. (2015). Smoking and life expectancy among HIV-infected individuals on antiretroviral therapy in Europe and North America. AIDS (London, England), 29(2), 221-229. https://doi.org/10.1097/ QAD.0000000000000540.

26. Bor, J., Herbst, A. J., Newell, M. L., \& Bärnighausen, T. (2013). Increases in adult life expectancy in rural South Africa: valuing the scale-up of HIV treatment. Science (New York, N.Y.), 339(6122), 961-965. https://doi.org/10.1126/science.1230413.

27. Sabin C. A. (2013). Do people with HIV infection have a normal life expectancy in the era of combination antiretroviral therapy? BMC medicine, 11, 251. https://doi.org/10.1186/1741-7015-11251.

28. Jia, H., Zack, M. M., Gottesman, I. I., \& Thompson, W. W. (2018). Associations of Smoking, Physical Inactivity, Heavy Drinking, and Obesity with Quality-Adjusted Life Expectancy among US Adults with Depression. Value in health : the journal of the International Society for Pharmacoeconomics and Outcomes Research, 21(3), 364-371. https://doi.org/10.1016/j. jval.2017.08.002. 